Establishing the Rationale for, and Practice of, User Research at the Humber Mental Health Teaching Trust

Graham Shields ${ }^{1}$, Mike Walsh ${ }^{1}$

${ }^{1}$ Humber Mental Health Services NHS Teaching Trust 


\title{
Establishing the rationale for, and practice of, service user research at the Humber Mental Health Teaching Trust
}

\author{
Graham H. Shields MSc with Mike Walsh
}

\begin{abstract}
User research in mental health should be seen as a separate specialism in a "hierarchy of emergence" and this is argued for with reference to the metaphor of a car crash that has a multiplicity of perspectives. Technical language and approaches are not always sufficient: proximity to our conditions may increase the accuracy of interpretation. The piece then describes the quest to establish user research at the Humber Mental Health Teaching Trust and points out the importance of determination by Trusts to provide resources and facilitation. In the process of this, quality criteria for assessing user research projects are briefly presented.
\end{abstract}

Service user research in the mental health field presents a challenge to traditional, reductionist methodologies that are often unable to acknowledge the validity of multiple, competing versions of understandings of the same phenomenon. After establishing a case for such an approach, I will describe aspects of our experiences in attempting to establish research by the Humber Mental Health Teaching NHS Trust.

Keywords: Service user research, facilitation and support, service quality

Imagine a car accident. The driver's wife is killed outright but he, along with his son and dog survive unscathed.

The police attend and take a few measurements and interviews. They write a formal, technical report for the judge quoting information about speed, skid length and a surmised order of events. They do not comment on the man's feelings about his wife or analyse in depth the trauma of the son in losing his mother, nor do they comment on the interpretation of the accident from the perspective of the dog. The language of the report is accessible to lawyers not laymen or animals, and is totally useless for the man's purposes of communicating his experience and meaning to his friends at the local, or for the son in explaining the network of events and meanings to his friends at school, or for the dog.

Each observer has a valid perspective on the accident and each is valid in helping to build a full picture of the event. Experts look at truth through the lenses of their specialism: it is perhaps impossible for them to detach from the presuppositions that influence the colours they observe. And how can humanity properly enter into the experience of the dog let alone communicate its findings in a way which dogs understand?

Systems thinking uses the terms 'hierarchy' and 'emergence' to express the truth that differing perspectives on a real situation can generate different levels of truth, (Checkland, P. 1981), such as in the above example of the crash. Things hidden, or totally unexpected, at one level of analysis become apparent at a higher, or lower, level of scrutiny. The order of 
hierarchy is determined by the extent to which the method of approach breaks down the problem into its component parts: geography is a higher level than environmental science for it looks at the consequences of largescale activity at a lower level without, necessarily, having to look at the lower level. In studying humanity, one may address the subject from the perspective of the theologian, the sociologist, or the biologist amongst others and each perspective reveals specific truths emergent from that level. For example, if studying a disease the theologian might draw on Biblical understandings, such as those expressed in the Book of Job, and ask questions about why we suffer and possibly blame Satan; the sociologist may look at the social inequalities which have created the conditions for the disease and blame the environment, whilst the biologist will refer to the functionality of various micro-organisms and blame bacteria. Each specialism on its own does not have the whole picture and cannot be seen as having a monopoly on the truth: versions of the truth do not have to be mutually exclusive. Satan may well have caused the illness through encouraging greed which led to social inequality and poverty, which resulted in a lack of cleanliness that led to a multiplication of bacteria resulting in infection.

Service user research, which draws on the particular perspective of those who actually experience mental illness, has the potential to observe emergent realities in a similar way to an academic specialism. It is an attempt to permit expression of the under-represented perspective of the service user and should not be seen as a source of competing truth but as a missing source of part of the truth. It addresses the power imbalances that exist between experts and their clients: it enables the dog to communicate on its own terms and study the problem within its own frame of reference as well as allowing the driver and son to express issues, such as those of meaning, which may not be fully looked at by traditional professionals.

To call the service user a dog is not meant to be pejorative but a metaphor for the power relationship between the system and the service user. The majority of service users would not be able to follow detailed arguments about brain chemistry but are expected to receive treatment from psychiatrists based on them. However, the majority of psychiatrists, medical scientists and care providers do not have personal experience of our conditions. Peter Beresford addresses the issue of distance between the phenomenon of mental illness and its interpretation and suggests a hypothesis in contradiction to the distance-objectivity required by the traditional scientific method as follows.

'The shorter the distance there is between direct experience and its interpretation (as for example can be offered by service user involvement in research and particularly service user controlled research), then the less distorted, inaccurate and damaging resulting research is likely to be.' Beresford, P. 2005:7

Service users are better placed to research and understand mental illness particularly in terms of its meanings and the efficacy of various treatments. That we can observe and discover for ourselves should be seen as a separate emergent layer in the hierarchy of knowledge. 
But service users also have a need to understand and sometimes contest knowledge accepted by various authorities. This requires service users to educate themselves and to be allowed to experiment to permit the owning or disowning of alleged truths. It requires the co-operation of those with the knowledge to share it in ways we can understand and to permit research, not only for purposes of gaining special additional knowledge from the perspective of the service user specialism, but to enable knowledge validation. Of course, there is an overlap in these two forms of service user research.

At the Humber Mental Health Teaching NHS Trust an attempt has been made to promote and establish service user research. The principal lesson we have learnt is that effective facilitation and support by experienced researchers appears essential.

Tony Hostick, Clinical Effectiveness Manager, imaginatively initiated the project and envisaged a self-run project with its own budget for research. A panel of nine met bi-monthly for about a year and comprised six service user and carer representatives, some with experience of research, and three employees of the Trust. We had the following aims:

- To establish the kind of research which service users could undertake locally.

- To look at ways of prioritising and commissioning research.

- To commission research projects.

- To establish links with other relevant organisations.

- To generate interest amongst service users and create a list of those willing to be involved.

- To provide for the needs of those involved by means of training and payment provision.

Whilst we had some success in achieving these, we needed: Concrete examples of existent service user research which we could attempt to emulate. We needed to look at real research.

1. Though we had some good facilitation, we had problems defining exactly what we were doing and we became fractious as a result; consequently, the style of undertaking that we were using was not really appropriate. The Trust needed to decide and act on our deliberations and even decide what had been decided and have an active determination to professionally initiate real projects.

2. Too much of our activity was concerned with measuring quality of research for the purposes of allocating resources. Whilst we were successful in generating appropriate criteria, we needed help to avoid having the quality tail wag the research dog. Our criteria were as follows.

- Service user run? Is the research put together by service users? Will the research be partially or wholly performed by service users?

- Support? Are the service users adequately advised and supported? 
- Methodology? Is the research well planned? Does it make sense? Are there any possible sources of bias not considered? Could it be done a better way?

- Use of Research? Will the conclusions be meaningful and relevant? Will the research be listened to by managers? Is it likely to lead to changes in services or attitudes? Will it be taken on board by service user groups for campaign purposes?

- Research Capacity? Does this proposal originate from a new group of service users? Does this research significantly improve the experience and knowledge of the research group?

- Costings? Has the research been adequately costed? Are the figures reasonable?

One of the authors gave a presentation of our achievements at the Involve conference, November 2004.

The panel no longer meets but a service user project stemming from the panel's work is undertaking research into service users' understandings of faith, belief and their interaction with mental illness. If the Trust had had a stronger determination as a whole organisation to provide resources, and had not had to weigh our project's needs against other demands, the possibility of being involved in research and its relevance could have been more actively promoted amongst service users.

Service user research is important to help establish a full picture of mental health conditions and services and to help the service user community own good research by others. For it to be a reality, it needs the support of NHS Trusts by means of the provision of active and decisive facilitation.

\section{References}

Beresford, P. 2005. Developing the theoretical basis for service user/survivor-led research and equal involvement in research. Epidemiologia e Psichiatria Sociale 14(1) pp. 4-9

Checkland, P. 1981. Systems Thinking, Systems Practice. John Wiley \& Sons Ltd, pp.74-82 